PROCEEDINGS OF THE

AMERICAN MATHEMATICAL SOCIETY

Volume 128, Number 6, Pages 1665-1672

S 0002-9939(99)05491-X

Article electronically published on October 18, 1999

\title{
UNIQUENESS OF THE LEAST-ENERGY SOLUTION FOR A SEMILINEAR NEUMANN PROBLEM
}

\author{
MASSIMO GROSSI
}

(Communicated by Lesley M. Sibner)

Abstract. We prove that the least-energy solution of the problem

$$
\begin{cases}-d \Delta u+u=u^{p} & \text { in } B, \\ u>0 & \text { in } B, \\ \frac{\partial u}{\partial \nu}=0 & \text { on } \partial B,\end{cases}
$$

where $B$ is a ball, $d>0$ and $1<p<\frac{N+2}{N-2}$ if $N \geq 3, p>1$ if $N=2$, is unique (up to rotation) if $d$ is small enough.

\section{INTRODUCTION}

In this paper we consider the following problem:

$$
\begin{cases}-d \Delta u+u=u^{p} & \text { in } \Omega, \\ u>0 & \text { in } \Omega, \\ \frac{\partial u}{\partial \nu}=0 & \text { on } \partial \Omega,\end{cases}
$$

where $\Omega$ is a bounded smooth domain in $\mathbb{R}^{N}, \nu$ is the unit outer normal to $\partial \Omega$, $d>0$ and $1<p<\frac{N+2}{N-2}$ if $N \geq 3, p>1$ if $N=2$.

To our knowledge the first existence result to (0.1) is due to Lin, Ni and Takagi (see [LNT]). In this paper the authors consider the functional

$$
J_{d}(u)=\frac{1}{2} \int_{\Omega}\left(d|\nabla u|^{2}+u^{2}\right)-\frac{1}{p+1} \int_{\Omega}\left(u^{+}\right)^{p+1}
$$

and

$$
c_{d}=\inf _{h \in \Gamma} \max _{t \in[0,1]} J_{d}(h(t))
$$

where $\Gamma=\left\{f \in C\left([0,1], H^{1}(\Omega)\right) \mid f(0)=0, f(1)=e\right\}$ and $e \neq 0$ is a non-negative function of $H^{1}(\Omega)$ such that $J_{d}(e)=0$. From the mountain-pass theorem (see [AR]) we have that $c_{d}$ is a positive critical value of $J_{d}$. Moreover, it turns out that $c_{d}$ is the least positive critical value for $J_{d}$ (see [LNT] or [NT]).

Therefore, we define a critical point $u_{d}$ of $J_{d}$ satisfying $J_{d}\left(u_{d}\right)=c_{d}$ a least-energy solution of (0.1).

Received by the editors July 9, 1998.

1991 Mathematics Subject Classification. Primary 35J70.

Key words and phrases. Uniqueness results, semilinear elliptic equations, Neumann problem.

This research was supported by M.U.R.S.T. (Project "Metodi Variazionali ed Equazioni Differenziali Non Lineari"). 
Clearly (0.1) admits the trivial solutions $u \equiv 1$ and $u \equiv 0$. In [LNT] it was proved that $c_{d}=O\left(d^{N / 2}\right)$ as $d \rightarrow 0$ and from this $0<J_{d}\left(u_{d}\right)<J_{d}(1)$, i.e. $u_{d}$ is a nonconstant solution provided $d$ is small enough.

Another important result concerns the shape of the least-energy solution $u_{d}$. In NT] it was shown that $u_{d}$ has only one local maximum in $\bar{\Omega}$, and it is achieved at exactly one point which lies on the boundary of $\Omega$, provided $d$ is small enough.

In this paper we will prove the uniqueness of the least-energy solution if $\Omega$ is a ball. Of course, in this case, if $u(x)$ is a solution of (10.1), then also $u(T x)$ solves (0.1) for any $T$ belonging to the orthogonal group $O(N)$; therefore by uniqueness we mean that any two solutions can be obtained from each other using the action of $O(N)$.

Theorem 1. Let us consider the problem (0.1) when $\Omega$ is a ball. Then there exists a $d_{0}>0$ such that for any $d<d_{0}$ the least-energy solution of (0.1) is unique.

We want to point out that uniqueness results for (0.1) cannot be obtained if we do not restrict the class of the solutions of [0.1). Indeed, in [DY] Dancer and Yan prove the existence of $k$-peak solutions to (0.1) (solutions with more than one local maximum point). Clearly the energy of these solutions is strictly greater than $c_{d}$. We mention here the papers of [BDS], Gu, W1], and [W2] for the existence of multipeak solutions in other domains.

The paper is organized as follows: in Section 1 we recall some important results which we use in the proof of Theorem [1. In Section 2 we prove Theorem 1

\section{KNOWN RESULTS}

In this section we recall some known facts which will be useful in the proof of Theorem 1 Here $\Omega$ is an arbitrary bounded smooth domain of $\mathbb{R}^{N}$ and $p$ is a real number such that $1<p<\frac{N+2}{N-2}$ if $N \geq 3$ and $p>1$ if $N=2$. We start by recalling some results on the existence and the shape of the least-energy solution $u_{d}$.

Theorem 1.1. Let $p>1$ if $N=1$ or 2 and let $1<p<\frac{N+2}{N-2}$ if $N \geq 3$. Then there exists a $d_{0}>0$ such that, for any $d<d_{0}$, (0.1) has a positive nonconstant solution $u_{d}$ satisfying

$$
\begin{gathered}
J_{d}\left(u_{d}\right)=c_{d} \\
\int_{\Omega} u_{d}^{q} \leq C_{1}(q) d^{N / 2} \quad \text { for any } q \geq 1,
\end{gathered}
$$

and

$$
\sup _{\Omega} u_{d}(x) \leq C_{2}
$$

with $C_{1}(q), C_{2}$ independent of $d$.

Proof. See $[\mathrm{LNT}$.

Theorem 1.2. Let $u_{d}$ be a least-energy solution to (1.3). Then $u_{d}$ has at most one local maximum in $\Omega$ and it is achieved exactly at one point which must lie on the boundary, provided d is sufficiently small.

Proof. See [NT], Theorem 2.1. 
Theorem 1.3. There is exactly one solution (up to translation) of the problem

$$
\begin{cases}-\Delta u+u=u^{p} & \text { in } \mathbb{R}^{N}, \\ u>0 & \text { in } \mathbb{R}^{N}, \\ u(x) \rightarrow 0 & \text { as }|x| \rightarrow \infty .\end{cases}
$$

Proof. See [K].

Next let us introduce a diffeomorphism which straightens a boundary portion of $\Omega$ as follows. We denote by $B_{R}$ the ball of $\mathbb{R}^{N}$ centered at the origin and radius $R$. Through translation and rotation of the coordinate system we may assume that $P$ is the origin and the inner normal to $\partial \Omega$ at $P$ is pointing in the direction of the positive $x_{N}$-axis. Then there exists a smooth function $\psi\left(x^{\prime}\right), x^{\prime}=\left(x_{1}, \ldots, x_{N-1}\right)$, defined for $|x|<\delta_{0}$ such that $\psi(0)=0, \nabla \psi(0)=0$ and

$$
\partial \Omega \cap \mathcal{N}=\left\{x=\left(x^{\prime}, x_{N}\right) \mid x_{N}=\psi\left(x^{\prime}\right)\right\}
$$

and

$$
\Omega \cap \mathcal{N}=\left\{x=\left(x^{\prime}, x_{N}\right) \mid x_{N}>\psi\left(x^{\prime}\right)\right\} .
$$

For $|y|<\delta^{\prime}$ let us define $x=\Phi(y)$, with $\Phi(y)=\left(\Phi_{1}(y), \ldots, \Phi_{N}(y)\right)$, by

$$
\Phi_{j}(y)= \begin{cases}y_{j}-y_{N} \frac{\partial \psi}{\partial x_{j}}\left(y^{\prime}\right) & \text { for } j=1, \ldots, N-1, \\ y_{N}+\psi\left(y^{\prime}\right) & \text { if } j=N .\end{cases}
$$

Since $\nabla \psi(0)=0$ the differential map $D \Phi$ of $\Phi$ satisfies $D \Phi(0)=I$, the identity map. Thus there exists the inverse map $y=\Phi^{-1}(x)$ between $C \subset \mathbb{R}^{N}$ and $B(0, \delta)$ with $\delta<\delta^{\prime}$.

Theorem 1.4. Let $u_{d}$ be a least-energy solution to (0.1) and let us denote by $P_{d}$ the point where the maximum of $u_{d}$ is achieved. Then for any $\epsilon>0$ there exist a positive constant $d_{0}$ and a subdomain $\Omega_{d}^{(i)} \subset \Omega$ such that for $\left.d \in\right] 0, d_{0}$ [ the following statements hold:

$$
\begin{gathered}
P_{d} \in \partial \Omega_{d} \quad \text { and } \quad \operatorname{diam}\left(\Omega_{d}^{(i)}\right) \leq C \sqrt{d}, \\
u_{d}(\Phi(\sqrt{d} y)) \rightarrow U(y) \text { in } C_{\mathrm{loc}}^{2}\left(\mathbb{R}_{+}^{N}\right) \text { as } d \rightarrow 0, \\
\left|u_{d}(x)\right| \leq C_{1} \epsilon e^{-\frac{\mu_{1} \delta(x)}{\sqrt{d}}} \quad \text { for } \quad x \in \Omega \backslash \Omega_{d}^{(i)},
\end{gathered}
$$

where $\delta(x)=\min \left\{\operatorname{dist}\left(x, \partial \Omega_{d}\right), \eta_{0}\right\}, C, C_{1}, \mu_{1}, \eta_{0}$ are positive constants depending only on $\Omega$ and $U(x)$ is the unique solution of (1.4) with maximum achieved at $P_{0}=\lim _{d \rightarrow 0} P_{d}$.

Proof. See [NT, (i) and (iii) of Theorem 2.3 and (4.29).

Theorem 1.5. Let us consider the problem

$$
\left\{\begin{array}{l}
-\Delta z+z=p U(x)^{p-1} z \quad \text { in } \mathbb{R}^{N}, \\
\|z\|_{L^{\infty}\left(\mathbb{R}^{N}\right)} \leq C,
\end{array}\right.
$$

where $U(x)$ is the unique solution of (1.4) symmetric with respect to the origin. Then the only nontrivial solutions of (1.9) are given by

$$
z=\sum_{i=1}^{N} \alpha_{i} \frac{\partial U}{\partial x_{i}}, \quad \alpha_{i} \in \mathbb{R}
$$

Proof. This result is contained in the proof of Proposition 1 of [D]. 


\section{The Uniqueness Result}

In this section we denote by $x=\left(x^{\prime}, x_{N}\right) \in \mathbb{R}^{N-1} \times \mathbb{R}$ a generic point of $\mathbb{R}^{N}$. Let us assume that $\Omega=B=\left\{\left.x \in \mathbb{R}^{N}|| x^{\prime}\right|^{2}+\left(x_{N}-1\right)^{2}<1\right\}$. From Theorem 1.2 the maximum of a least-energy solution $u$ of $(0.1)$ is achieved on the boundary of $B$ if $d$ is small enough. So, since the problem (0.1) is invariant with respect to the orthogonal group of $\mathbb{R}^{N}$, by a suitable rotation we can assume that $u$ attains its maximum at the origin. Hence $\nabla u(0)=0$ for any least-energy solution of (0.1).

We suppose by contradiction that there exist a sequence $d_{n} \searrow 0$ and two distinct least-energy solutions $u_{1, n}$ and $u_{2, n}$ which solve (0.1) with $d=d_{n}$. So we can suppose that $u_{1, n}$ and $u_{2, n}$ achieve their maximum at the origin for any $n \in \mathbb{N}$. Since $u_{1, n}-u_{2, n} \not \equiv 0$ we can consider

$$
z_{n}(x)=\frac{u_{1, n}(x)-u_{2, n}(x)}{\left\|u_{1, n}-u_{2, n}\right\|_{L^{\infty}}} .
$$

By assumption on $u_{1, n}$ and $u_{2, n}$ we have

$$
\nabla z_{n}(0)=0 .
$$

Note that $z_{n}$ satisfies

$$
\begin{cases}-d_{n} \Delta z_{n}+z_{n}=c_{n}(x) z_{n} & \text { in } B \\ \frac{\partial z_{n}}{\partial \nu}=0 & \text { on } \partial B \\ \left\|z_{n}\right\|_{L^{\infty}(B)}=1, & \end{cases}
$$

where

$$
c_{n}(x)=p \int_{0}^{1}\left(t u_{1, n}(x)+(1-t) u_{2, n}(x)\right)^{p-1} d t .
$$

Let us set

$$
\widetilde{z}_{n}(x)=z_{n}\left(\sqrt{d_{n}} x\right), \quad \widetilde{z}_{n}: B / \sqrt{d_{n}} \mapsto \mathbb{R} .
$$

We remark that for any compact set $K \subset \mathbb{R}_{+}^{N}$, where $\mathbb{R}_{+}^{N}=\left\{x \in \mathbb{R}^{N} \mid x_{N}>0\right\}$, we have that $K \subset B / \sqrt{d_{n}}$ for $n$ large enough. In the following proposition we study the asymptotic behavior of $\widetilde{z}_{n}$.

Proposition 2.1. We have that

$$
\widetilde{z}_{n} \rightarrow z \quad \text { in } C_{\mathrm{loc}}^{1}\left(\mathbb{R}_{+}^{N}\right),
$$

where $z$ is a bounded solution of

$$
-\Delta z+z=p U^{p-1} z \quad \text { in } \mathbb{R}^{N}
$$

and $U$ is the unique solution of (1.4) symmetric with respect to the origin.

Proof. Let us consider the diffeomorphism $\Phi$ between $B(0, \delta)$ and $C \subset \mathbb{R}^{N}$ which straightens the boundary of $B$ (see (1.5)). Now, as in [NT], let us set

$$
v_{n}(y)=z_{n}(\Phi(y)), \quad y \in \overline{B_{2 k}^{+}},
$$

where $B_{2 k}^{+}=\left\{y \in B_{2 k} \mid y_{N}>0\right\}$.

Step 1. In this step we prove that

$$
v_{n}\left(\sqrt{d_{n}} y\right) \rightarrow z(y) \quad \text { in } C_{\mathrm{loc}}^{2}\left(\mathbb{R}_{+}^{N}\right) .
$$


First of all we extend $v_{n}$ to $B(0,2 k)$ by reflection,

$$
\widetilde{v}_{n}(y)= \begin{cases}v_{n}(y) & \text { if } y \in \overline{B_{2 k}^{+}} \\ v_{n}\left(y^{\prime},-y_{N}\right) & \text { if } y \in \overline{B_{2 k}^{-}}\end{cases}
$$

with $\overline{B_{2 k}^{-}}=\left\{y \in B_{2 k} \mid y_{N}<0\right\}$. Moreover we define a scaled function $w_{n}(z)$ by

$$
w_{n}(y)=\widetilde{v}_{n}\left(\sqrt{d_{n}} y\right) \quad \text { for } y \in \bar{B}_{\frac{k}{\sqrt{d_{n}}}} .
$$

It is easily seen that

$$
w_{n} \in C^{2}\left(\bar{B}_{\frac{k}{\sqrt{d_{n}}}} \backslash\left\{y_{N}=0\right\}\right) \cap C^{1}\left(\bar{B}_{\frac{k}{\sqrt{d_{n}}}}\right)
$$

since $\frac{\partial v_{n}}{\partial y_{N}}$ on $y_{N}=0$ and that $w_{n}$ satisfies the elliptic equation

$$
-L_{n} w_{n}+w_{n}=\widetilde{c}_{n}(y) w_{n}
$$

with

$$
L_{n} w_{n}=\sum_{i, j=1}^{N} a_{i j}^{n}(y) \frac{\partial^{2} w_{n}}{\partial z_{i} \partial z_{j}}+\sqrt{d_{n}} \sum_{j=1}^{N} b_{j}^{n}(y) \frac{\partial w_{n}}{\partial z_{j}}
$$

in $B_{\frac{k}{\sqrt{d_{n}}}} \backslash\left\{y_{N}=0\right\}$, where $a_{i j}^{n}, b_{j}^{n}$ and $\widetilde{c}_{n}(y)$ are defined as follows. First, put

$$
\begin{gathered}
\Psi=\Phi^{-1} \\
a_{i j}(y):=\sum_{l=1}^{N} \frac{\partial \Psi_{i}}{\partial x_{l}}(\Phi(y)) \frac{\partial \Phi_{j}}{\partial x_{l}}(\Phi(y)), \quad 1 \leq i, j \leq N \\
b_{j}(y):=\left(\Delta \Psi_{j}\right)(\Phi(y)), \quad j=1, \ldots, N \\
\hat{c}_{n}(y)=c_{n}(\Phi(y)) .
\end{gathered}
$$

Then set

$$
\begin{aligned}
& a_{i j}^{n}(y):= \begin{cases}a_{i j}\left(\sqrt{d_{n}} y\right) & \text { if } y_{N} \geq 0 \\
(-1)^{\delta_{N}^{i}}+\delta_{N}^{j} a_{i j}\left(\sqrt{d_{n}} y^{\prime},-\sqrt{d_{n}} y_{N}\right) & \text { if } y_{N}<0\end{cases} \\
& b_{j}^{n}(y):= \begin{cases}b_{j}\left(\sqrt{d_{n}} y\right) & \text { if } y_{N} \geq 0, \\
(-1)^{\delta_{N}^{j}} b_{j}\left(\sqrt{d_{n}} y^{\prime},-\sqrt{d_{n}} y_{N}\right) & \text { if } y_{N}<0,\end{cases} \\
& \widetilde{c}_{n}(y):= \begin{cases}\hat{c}_{n}\left(\sqrt{d_{n}} y\right) & \text { if } y_{N} \geq 0, \\
(-1)^{\delta_{N}^{j}} \hat{c}_{n}\left(\sqrt{d_{n}} y^{\prime},-\sqrt{d_{n}} y_{N}\right) & \text { if } y_{N}<0,\end{cases}
\end{aligned}
$$

where $\delta_{j}^{i}$ is the Kroneker symbol.

Note that by (1.3) the functions $w_{n}$ and $c_{n}$ are uniformly bounded with respect to $n$. Then, by using the standard $L^{r}$-estimate and the interior Schauder estimate as in [NT], pp. 834-836, we obtain that

$$
w_{n} \rightarrow z \quad \text { in } C_{\mathrm{loc}}^{2}\left(\mathbb{R}^{N}\right)
$$

and

$$
-L_{n} w_{n} \rightarrow-\Delta z
$$

Hence, since by (1.7) and (2.4) we get $\widetilde{c}_{n} \rightarrow p U^{p-1}, z$ is a solution of

$$
-\Delta z+z=p U^{p-1} z \quad \text { in } \mathbb{R}^{N} .
$$

By the definition of $w_{n}$ and $\widetilde{v}_{n}$ (2.8) follows. 
Step 2. In this step we prove the claim of the proposition.

The function $\widetilde{z}_{n}$ satisfies the equation

$$
\begin{cases}-\Delta \widetilde{z}_{n}+\widetilde{z}_{n}=c_{n}\left(\sqrt{d_{n}}\right) \widetilde{z}_{n} & \text { in } B / \sqrt{d_{n}} \\ \widetilde{z}_{n}>0 & \text { in } B / \sqrt{d_{n}} \\ \frac{\partial \widetilde{z}_{n}}{\partial \nu}=0 & \text { on } \partial B / \sqrt{d_{n}} .\end{cases}
$$

From (1.3) we get $\left\|c_{n} \widetilde{z}_{n}\right\|_{\infty} \leq C$ and then by standard $L^{p}$-estimates we deduce, for a subsequence, $\left\|\widetilde{z}_{n}\right\|_{C^{1}(K)} \leq C$ for any compact set $K \subset \mathbb{R}^{N}$.

By Step 1 we know that $\left\|z_{d_{n}}\left(\Phi\left(\sqrt{d_{n}} y\right)\right)-U(y)\right\|_{C_{\mathrm{loc}}^{0}\left(\mathbb{R}_{+}^{N}\right)} \rightarrow 0$. So if we show that

$$
\left\|z_{d_{n}}\left(\Phi\left(\sqrt{d_{n}} y\right)\right)-\widetilde{u}_{n}(y)\right\|_{C_{\mathrm{loc}}^{0}\left(\mathbb{R}_{+}^{N}\right)} \rightarrow 0
$$

the claim follows.

Let us fix a compact set $K \subset \mathbb{R}^{N}$. By the definition of $\Phi$ (see (1.5)) we get

$$
\left|\Phi\left(\sqrt{d_{n}} y\right)-\sqrt{d_{n}} y\right|^{2}=\left.\left|d_{n} y_{N}^{2}\right| \nabla_{y^{\prime}} \psi\left(\sqrt{d_{n}} y\right)\right|^{2}+\psi^{2}\left(\sqrt{d_{n}} y\right) \mid \rightarrow \psi^{2}(0)=0
$$

Then, since $\left|\nabla u_{d_{n}}\right| \leq C$ in $K$, we obtain that

$$
\left\|z_{d_{n}}\left(\Phi\left(\sqrt{d_{n}} y\right)\right)-\widetilde{z}_{n}(y)\right\|_{C^{0}(K)} \leq C|| \Phi\left(\sqrt{d_{n}} y\right)-\sqrt{d_{n}} y \|_{C^{0}(K)} \rightarrow 0,
$$

and this finishes the proof.

Proof of Theorem 1. Let us again consider the functions $z_{n}$ and $\widetilde{z}_{n}$ defined by (2.1) and (2.5). First of all we remark that since $u_{1, n}$ and $u_{2, n}$ solve (0.1) we have

$$
\int_{\Omega} u_{1, n} u_{2, n}\left(u_{1, n}^{p-1}-u_{2, n}^{p-1}\right)=0
$$

and then $\widetilde{z}_{n}$ does change sign.

Since we have that $\left\|\widetilde{z}_{n}\right\|_{L^{\infty}\left(B / \sqrt{d_{n}}\right)}=1$ we can assume that there exists a sequence of points $\widetilde{x}_{n} \in B / \sqrt{d_{n}}$ such that $\widetilde{z}_{n}\left(\widetilde{x}_{n}\right)=\max _{x \in B / \sqrt{d_{n}}} \widetilde{z}_{n}(x)=1$. Moreover we have that

$$
\nabla \widetilde{z}_{n}\left(\widetilde{x}_{n}\right)=0 \quad \text { and } \quad \Delta \widetilde{z}_{n}\left(\widetilde{x}_{n}\right) \leq 0
$$

(this is obvious if $\widetilde{x}_{n} \in B / \sqrt{d_{n}}$ and it follows by $\frac{\partial \widetilde{z}_{n}}{\partial \nu}\left(\widetilde{x}_{n}\right)=0$ if $\left.\widetilde{x}_{n} \in \frac{\partial B}{\sqrt{d_{n}}}\right)$.

From Proposition 2.1 the following alternative occurs: either

$$
\widetilde{z}_{n} \rightarrow 0
$$

or

$$
\widetilde{z}_{n} \rightarrow \sum_{i=1}^{N} \alpha_{i} \frac{\partial U}{\partial x_{i}} \quad \text { for some }\left(\alpha_{1}, \ldots, \alpha_{N}\right) \in \mathbb{R}^{N} \backslash\{0\} .
$$

We will prove that in any case we reach a contradiction.

Case 1. $\left|\widetilde{z}_{n}\right| \rightarrow z \equiv 0$.

Here we have the following alternative:

i) $\left|\widetilde{x}_{n}\right| \leq C$

ii) $\left|\widetilde{x}_{n}\right| \rightarrow \infty$. 
Case i). $\left|\widetilde{x}_{n}\right| \leq C$.

Let $x_{0}=\lim _{n} \widetilde{x}_{n}$. If $x_{0} \notin \partial \mathbb{R}_{+}^{N}$, the claim follows easily. If $x_{0} \in \partial \mathbb{R}_{+}^{N}$, we again consider the diffeomorphism $\Phi$ between $B\left(0, \delta^{\prime}\right)$ and $B(0, \delta)$ which straightens the boundary. Since $\widetilde{x}_{n} \in B / \sqrt{d_{n}}$ we have that $\widetilde{x}_{n}=\frac{x_{n}}{\sqrt{d_{n}}}$ with $x_{n} \in B$ and $x_{n} \rightarrow 0$. Then there exists $P_{n} \in B_{\delta}^{+}$such that

$$
\Phi\left(P_{n}\right)=x_{n} .
$$

Set $y_{n}=\frac{P_{n}}{\sqrt{d_{n}}}$. Then, since $\Phi(0)=0$ and $\Phi$ is a diffeomorphism in $B_{\delta}^{+}$,

$$
\left|\sqrt{d_{n}} y_{n}\right|=\left|P_{n}\right|=\left|\Phi^{-1}\left(x_{n}\right)\right|=\left|\Phi^{-1}\left(\sqrt{d_{n}} \widetilde{x}_{n}\right)\right| \leq C\left|\sqrt{d_{n}} \widetilde{x}_{n}\right|,
$$

so $\left|y_{n}\right| \leq C$. Finally, by (2.7)-(2.9)

$$
1=\widetilde{z}_{n}\left(\widetilde{x}_{n}\right)=z_{n}\left(x_{n}\right)=z_{n}\left(\Phi\left(P_{n}\right)\right)=z_{n}\left(\Phi\left(\sqrt{d_{n}} y_{n}\right)\right) \rightarrow 0
$$

and this gives a contradiction.

Case ii). $\left|\widetilde{x}_{n}\right| \rightarrow \infty$.

Let us prove that $c_{n}\left(\sqrt{d_{n}} \widetilde{x}_{n}\right) \rightarrow 0$. We have

$$
c_{n}\left(\sqrt{d_{n}} \widetilde{x}_{n}\right)=p \int_{0}^{1}\left(t u_{1, n}\left(\sqrt{d_{n}} \widetilde{x}_{n}\right)+(1-t) u_{2, n}\left(\sqrt{d_{n}} \widetilde{x}_{n}\right)\right)^{p-1} d t .
$$

Again since $\widetilde{x}_{n} \in B / \sqrt{d_{n}}$ there exists $x_{n} \in B$ such that $x_{n}=\sqrt{d_{n}} \widetilde{x}_{n}$ and

$$
\frac{\left|x_{n}\right|}{\sqrt{d_{n}}} \rightarrow \infty \text {. }
$$

Then (1.6) and (1.7) of Theorem 1.4 applies: since by (2.25) $x_{n} \notin B_{d_{n}}^{(i)}$ we obtain, for any $\epsilon>0$,

$$
u_{1, n}\left(x_{n}\right)<\epsilon \text { and } u_{2, n}\left(x_{n}\right)<\epsilon \text { for } n \text { large. }
$$

Hence $c_{n}\left(\sqrt{d_{n}} \widetilde{x}_{n}\right)=c_{n}\left(x_{n}\right) \rightarrow 0$. Finally from (2.19) we obtain

$$
0 \leq-\Delta \widetilde{z_{n}}\left(\widetilde{x}_{n}\right)=c_{n}\left(x_{n}\right)-1 \rightarrow-1,
$$

and this gives a contradiction.

Case 2. $\left|\widetilde{z}_{n}\right| \rightarrow z=\sum_{i=1}^{N} \alpha_{i} \frac{\partial U}{\partial x_{i}} \quad$ for some $\left(\alpha_{1}, \ldots, \alpha_{N}\right) \in \mathbb{R}^{N} \backslash\{0\}$.

First of all let us prove that

$$
\frac{\partial^{2} U}{\partial x_{j}^{2}}(0)<0 \quad \text { and } \quad \frac{\partial^{2} U}{\partial x_{i} \partial x_{j}}(0)=0 \text { if } i \neq j, i, j \in\{1, \ldots, N\} .
$$

This is a well-known fact (see [NT] for example), but we repeat the proof for the reader's convenience. Since the function $U(\rho)$ is radially decreasing for $\rho>0$ (see [GNN]), we have that $\frac{\partial U}{\partial x_{j}}=U^{\prime}(\rho) \frac{x_{j}}{\rho}>0$ in $T_{j}=\left\{x \in \mathbb{R}^{N} \mid x_{j}<0\right\}$. Hence $\left.\frac{\partial U}{\partial x_{j}}\right|_{\partial T_{j}} \equiv 0$ and then $\frac{\partial^{2} U}{\partial x_{i} \partial x_{j}}(0)=0$ for any $i \neq j$. Moreover $f=\frac{\partial U}{\partial x_{j}}$ solves

$$
\begin{cases}-\Delta f+f=p U(x)^{p-1} f & \text { in } T_{j}, \\ f>0 & \text { in } T_{j}, \\ f=\frac{\partial U}{\partial x_{j}}=0 & \text { on } \partial T_{j} .\end{cases}
$$


So, by Hopf's Lemma, $\frac{\partial f}{\partial x_{j}}=\frac{\partial^{2} U}{\partial x_{j}^{2}}(0)<0$ on $\partial T_{j}$, and then (2.28) is proved.

Now let us recall that $\nabla \widetilde{z}_{n}(0)=0$. Since $\Phi(0)=0$ we have

$$
\nabla\left(z_{n} \circ \Phi\right)(0)=0 .
$$

Then since $\left(\alpha_{1}, \ldots, \alpha_{N}\right) \neq(0, \ldots, 0)$ there exists $\alpha_{j} \neq 0$. Finally, from (2.8), (2.28) and $(2.29)$ we get

$$
0=\frac{\partial\left(z_{n} \circ \Phi\right)}{\partial x_{j}}(0) \rightarrow \sum_{i=1}^{N} \alpha_{i} \frac{\partial^{2} U}{\partial x_{i} \partial x_{j}}(0)=\alpha_{j} \frac{\partial^{2} U}{\partial x_{j}^{2}} \neq 0,
$$

a contradiction. The proof of Theorem 1 is finished.

\section{REFERENCES}

[AR] A. Ambrosetti and P. H. Rabinowitz, "Dual variational methods in critical point theory and applications", J Funct. Anal. 14, 349-381, (1973). MR 51:6412

[BDS] P. Bates, E.N. Dancer and J. Shi, "Multi-spike stationary solutions on the Cahn-Hilliard equation in higher dimension and instability", preprint.

[D] E.N. Dancer, "On the uniqueness of the positive solution of a singularly perturbed problem", Rocky Mountain Journal of Mathematics 25 (1995), 957-975. MR 96j:35021]

[DY] E.N. Dancer and S. Yan, "Multipeak solutions for a singularly perturbed Neumann problem" (to appear).

[GNN] B. Gidas, W.N. Ni and L. Nirenberg, "Symmetry of positive solutions of nonlinear elliptic

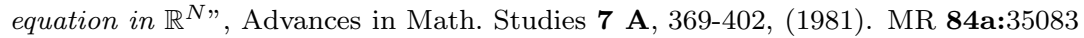

[GT] D. Gilbarg and N. Trudinger, "Elliptic partial differential equations of second order", Springer Verlag (1983). MR 86c:35035

[Gu] C.Gui, "Multipeak solutions for a semilinear Neumann problem", Duke Math. J. 84, 739769, (1996). MR 97i:35052

[K] M.K. Kwong, "Uniqueness of positive solutions of $\Delta u-u+u^{p}=0$ in $\mathbb{R}^{N}$ ", Arch. Rat. Mech. Anal., 105, 243-266, (1989). MR 90d:35015

[LN] C.S. Lin and W.M. Ni, "On the diffusion coefficient of a semilinear Neumann problem, Calculus of Variations and Partial Differential Equations", S. Hildebrandt, D. Kinderlehrer and M. Miranda, eds., Lecture Notes in Math. 1340, Springer-Verlag, Berlin-HeidelbergNew York-Tokyo, 160-174, (1988). MR 90d:35101

[LNT] C.S. Lin, W.M. Ni and I. Takagi, "Large amplitude stationary solutions to a chemotaxis system", J. Diff. Eqns. 72, 1-27, (1988). MR 89e:35075

[NT] W.M. Ni and I. Takagi, "On the shape of Least Energy Solutions to a Semilinear Neumann Problem", Comm. Pure Math. Appl., Vol XLIV, 819-851, (1991). MR 92i:35052

[W1] Z.Q. Wang, "On the existence of multiple single-peaked solution for a semilinear Neumann problem", Arch. Rat. Mech. Anal., 120, 375-399, (1992). MR 93k:35109

[W2] Z.Q. Wang, "Nonradial solutions of nonlinear Neumann problems in radially symmetric domains", Topology in Nonlinear analysis (Warsaw, 1994), 85-96, Banach Center Publications, 35, Polish Acad. Sci., Warsaw, (1996). MR 98e:35075

Dipartimento di Matematica, Università di Roma "La Sapienza", P.le A. Moro 2, 00185, Roma, Italy

E-mail address: grossi@mat.uniroma1.it 